hep-th/9907204

\title{
D3-brane shells to black branes on the Coulomb branch
}

\author{
Steven B. Giddings' and Simon F. Ross' \\ Department of Physics, \\ University of California \\ Santa Barbara, CA 93106-9530
}

\begin{abstract}
We use the AdS/CFT duality to study the special point on the Coulomb branch of $\mathcal{N}=4 S U(N)$ gauge theory which corresponds to a spherically symmetric shell of $D 3$-branes. This point is of interest both because the spacetime region inside the shell is flat, and because this configuration gives a very simple example of the transition between D-branes in the perturbative string regime and the nonperturbative regime of black holes. We discuss how this geometry is described in the dual gauge theory, through its effect on the two-point functions and Wilson loops. In the calculation of the two-point function, we stress the importance of absorption by the branes.
\end{abstract}

\footnotetext{
${ }^{1}$ giddings@physics.ucsb.edu

${ }^{2}$ sross@cosmic.physics.ucsb.edu
} 
The AdS/CFT correspondence [1] 3] allows us to study any asymptotically AdS geometry. Much work on this correspondence has focused on learning about the field theory from known properties of the supergravity, and hence has concentrated on the study of perturbations on a pure AdS background. However, to study the implications of the duality for gravitational physics, it is interesting to consider a larger class of spacetimes.

A particularly interesting set of questions in the AdS/CFT correspondence regards how the correspondence describes black holes. We would especially like to understand how to treat black hole formation, how to describe the observations of infalling observers and compare them with observations made outside, and finally how (or whether) the correspondence addresses issues of unitarity.

As an intermediate step on the path to such questions, one would like to consider the simpler problem of the collapse of a shell of three-branes to form a black brane. There are static shell solutions that are special cases of an interesting class of solutions corresponding to multi-center D3-brane geometries where the distribution of $D 3$-branes has some special symmetry; that is, to special points on the Coulomb branch of the gauge theory. Some geometries of this type were obtained in [4, as the extreme limits of families of rotating D3-brane metrics. Solutions with D3-branes distributed in disks of various dimensions were also studied in [5, 6], where the two-point function and Wilson loops in these backgrounds were discussed.

In the present paper, we will consider the geometry introduced in [4], where the D3branes lie in a spherically symmetric shell, so that the whole $S O(6)$ rotational symmetry of the pure $\mathrm{AdS}_{5} \times S^{5}$ space is preserved. In this case, the metric is

$$
d s^{2}=H^{-1 / 2}\left(-d t^{2}+d x_{\|}^{2}\right)+H^{1 / 2}\left(d r^{2}+r^{2} d \Omega_{5}^{2}\right),
$$

where $H=1+R^{4} / L^{4}$ for $r<L$, and $H=1+R^{4} / r^{4}$ for $r>L$. (Since the spacetime is static, one could equally well consider a Euclidean solution, but our main interest will be in the Lorentzian case.) In the near horizon limit, which is $r, L \ll R$ in these coordinates, the spacetime is the $\operatorname{AdS}_{5} \times S^{5}$ spacetime with curvature radius $R$ outside the shell, and flat spacetime inside the shell. A rescaling of the coordinates shows the near-horizon region includes the whole $\mathrm{AdS}_{5} \times S^{5}$ spacetime. This near-horizon region will be the main focus of this paper. Although the shell is at $r=L$, its physical radius is $R$. Note also that the shell is flat in the directions $x_{\|}$which are along the individual branes; this makes it possible to have a static configuration. We give the Penrose diagram for this spacetime in Fig. 1. Unlike the geometries considered in [5], there is no region of large curvatures in this spacetime. In fact, since the solution has no Weyl curvature, and the local geometry is maximally supersymmetric, there should be no $\alpha^{\prime}$ corrections at all, by the argument of [7]. The solution as a whole will preserve only half the supersymmetries; the other half are broken by the presence of the D3-branes. 


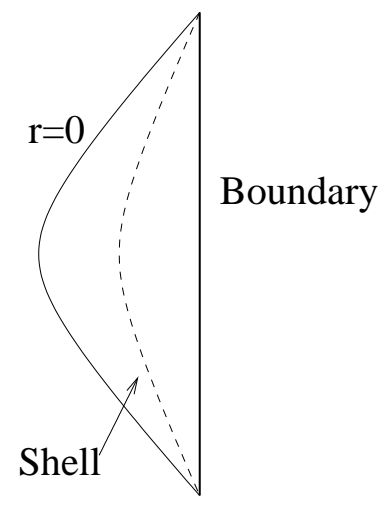

Figure 1: Penrose diagram of the near-horizon spacetime with a spherically symmetric shell.

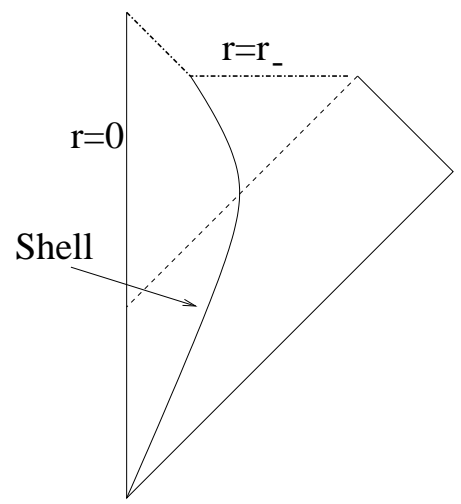

Figure 2: A proposed Penrose diagram for the collapse of a three-brane shell to form a non-extremal black three-brane. Note that the singularity forms at $r=r_{-}>0$.

If one gives this solution a "kick," it will collapse to form a non-extremal black brane, as shown in Fig. 2. We have not yet been able to analytically treat such solutions, so as a first step we will instead consider the physics of the one-parameter family of static solutions interpolating between large and small radius shells. This family provides a very simple example of a continuous path of configurations that connects the perturbative and non-perturbative regimes of string theory. In one limit the shell becomes widely separated $D 3$-branes, easily treated by perturbative string theory, and in the opposite limit the shell becomes a black brane with strong gravitational effects. It is here that the AdS/CFT correspondence and related ideas have suggested profound modifications to our semiclassical notions about black holes, and we are interested to get more hints about how such modifications arise.

Our interest in this solution has several other motivations. Firstly, the metric is 
almost as simple as pure AdS, being static and having the full rotational symmetry. This will make it possible to make analytic calculations. Secondly, it would seem to be interesting to study how the flat space region in this geometry is described in the gauge theory, in the hope that this might give more insight into the extension of the correspondence to asymptotically flat boundary conditions. (Unfortunately, we will not make much progress on this issue.) Furthermore, analyzing a geometry of this kind forces us to address the non-locality in the correspondence, since the geometry outside $r=L$ is exactly $\mathrm{AdS}_{5} \times S^{5}$, and it will therefore not signal its non-triviality in the one-point function $\langle\mathcal{O}\rangle$ of any operator dual to a supergravity field. Finally, these configurations pose a new challenge to the AdS/CFT duality: as we'll see, the supergravity predicts a spectrum of resonances that is not yet understood from the Yang-Mills perspective.

The statement regarding vanishing of the one-point functions might seem to be in conflict with the identification of this supergravity solution with a point on the Coulomb branch of the gauge theory, since the gauge theory would predict non-zero vacuum expectation values for the Higgs fields $X^{i}$ (giving the positions of the branes in the transverse space). However, the two points of view can be reconciled so long as none of the operators which should have non-zero vevs from the gauge theory point of view live in the short chiral primary multiplets whose dimensions are protected. $\left\langle\operatorname{tr}\left(X^{i}\right)\right\rangle$ will vanish by the spherical symmetry of the distribution. (At finite $N$, it may have a value of $O(1 / N)$, since the distribution doesn't have exact spherical symmetry; this corresponds to a quantum effect that will be invisible from our classical analysis.) An example of a non-vanishing vev is $\left\langle\operatorname{tr}\left(X^{i} X^{i}\right)\right\rangle \sim L^{2}$, but this operator is not a chiral primary, so it should correspond to some massive string excitation from the bulk point of view. It is interesting that the gauge theory makes a definite prediction for the value of this vev, but we were not able to use this to explore the connection between this spacetime and the gauge theory.

As already remarked in [8, 9], the presence of the shell will affect the two-point function in the gauge theory, and it should be possible to learn about how it is represented in the gauge theory by studying this two-point function. The main point of the present paper is to carry out this analysis of the two-point function, [3 and use it to probe the properties of the shell configuration and the transition from perturbative $D 3$-branes to configurations with macroscopic horizons. Another probe which will be sensitive to the presence of the shell is a Wilson loop, and we will briefly discuss the effect of the shell on the Wilson loops at the end of the paper.

According to the prescription of [2, 3], the generating function for correlators in the gauge theory is identified with the partition function of supergravity, with appropriately

\footnotetext{
${ }^{3}$ While this paper was in preparation, 10 appeared, which has a substantial overlap with our calculation. One important distinction is that we will include the effects of absorption by the shell.
} 
specified boundary conditions:

$$
\left\langle\exp i \int \phi_{0} \mathcal{O}\right\rangle=Z\left[\phi_{0}\right]
$$

At large $R=\left(g^{2} N\right)^{1 / 4}$ (we set $l_{s}=1$ ), we can thus calculate the two-point function of an operator dual to some test scalar field propagating in the background (1) in the semiclassical approximation for supergravity, by calculating the action for the scalar field subject to appropriate boundary conditions. For convenience, we will work in momentum space, so what we want are solutions to the ten-dimensional wave equation $\nabla^{2} \phi=0$ of the form

$$
\phi=e^{-i \omega t+i \vec{k} \cdot \vec{x}_{\|}} \Phi(r, \Omega) .
$$

Since the spacetime is spherically symmetric in the $S^{5}$, there will be no coupling between different partial waves. In the flat region $r<L$, the appropriate solution for $\Phi$ is

$$
\Phi(r, \Omega)=\frac{A}{r^{2}} J_{l+2}\left(q \frac{R^{2}}{L^{2}} r\right) Y_{l \vec{m}}(\Omega)
$$

where $q^{2}=\omega^{2}-k^{2}$. In the AdS region, the solution is

$$
\Phi(r, \Omega)=-\left(\frac{q}{2}\right)^{l+2} \frac{\pi}{(l+1) !} z^{2}\left(N_{l+2}(q z)+C_{l} J_{l+2}(q z)\right) Y_{l \vec{m}}(\Omega),
$$

where we have introduced the convenient coordinate $z=R^{2} / r$ in the AdS region. This solution behaves as $\phi \rightarrow z^{-l} e^{-i \omega t+i \vec{k} \cdot \vec{x}_{\|}} Y_{l \vec{m}}(\Omega)$ as $z \rightarrow 0$, which are the correct boundary conditions for it to give the bulk-boundary propagator $K_{B \partial}$.

If we neglect absorption by the shell, we should require that both $\phi$ and its derivative are continuous at the shell. This fixes the two constants of integration; in particular, it gives

$$
C_{l}=-\frac{J_{l+2}^{\prime}(s) N_{l+2}(s)+J_{l+2}(s) N_{l+2}^{\prime}(s)}{2 J_{l+2}(s) J_{l+2}^{\prime}(s)},
$$

where $s=q R^{2} / L$ is a natural dimensionless measure of the energy. As noted previously in [5, 6, [10], the energy scale associated with the shell from the supergravity point of view is $q \sim L / R^{2} \sim L / \sqrt{g N}$, rather than the much higher scale set by the Higgs vevs $q \sim L$. One suggestion for the physics responsible for this scale is that it arises from states consisting of W-boson-anti-W-boson pairs with large binding energy due to the large coupling [11].

For general boundary conditions

$$
\phi \rightarrow \int \frac{d^{4} k}{(2 \pi)^{4}} \sum_{l \vec{m}} \phi_{l \vec{m}}(k) z^{-l} e^{i k x} Y_{l \vec{m}}(\Omega),
$$


the action reduces to a surface term at infinity,

$$
\begin{aligned}
I & =\frac{1}{2} \int_{z=0} d \Sigma^{\mu} \phi \nabla_{\mu} \phi \\
& =\frac{R^{8}}{2} \int \frac{d^{4} k}{(2 \pi)^{4}} \sum_{l m} \frac{d^{4} k^{\prime}}{(2 \pi)^{4}} \sum_{l^{\prime} \vec{m}^{\prime}} \phi_{l \vec{m}}(k) \phi_{l^{\prime} \vec{m}^{\prime}}\left(k^{\prime}\right) \lim _{z \rightarrow 0} z^{-l-3} \partial_{z} K_{B \partial}\left(k, l, \vec{m} ; k^{\prime}, l^{\prime}, \vec{m}^{\prime} ; z\right) .
\end{aligned}
$$

This gives a two-point function

$$
\left\langle\mathcal{O}_{l \vec{m}}(\omega, \vec{k}) \mathcal{O}_{l^{\prime} \vec{m}^{\prime}}\left(\omega^{\prime}, \vec{k}^{\prime}\right)\right\rangle=-i R^{8} \lim _{z \rightarrow 0} z^{-l-3} \partial_{z} K_{B \partial}\left(k, l, \vec{m} ; k^{\prime}, l^{\prime}, \vec{m}^{\prime} ; z\right) .
$$

In evaluating the limit in this expression, there are singular terms which may be dropped as they give contact terms on the boundary. The final expression is

$$
\begin{aligned}
\left\langle\mathcal{O}_{l m}(\omega, k) \mathcal{O}_{l^{\prime} \vec{m}^{\prime}}\left(\omega^{\prime}, k^{\prime}\right)\right\rangle= & \frac{i R^{8}}{(l+1) !} \delta_{l l^{\prime}} \delta_{\vec{m} \vec{m}^{\prime}}(2 \pi)^{4} \delta^{4}\left(k-k^{\prime}\right)\left(\frac{q}{2}\right)^{2 l+4} \frac{l+4}{(l+2) !} \\
& \times\left\{2 \ln (q / 2)-[\psi(1)+\psi(l+3)]+2 /(l+4)+\pi C_{l}\right\} .
\end{aligned}
$$

Notice that this two-point function has poles, at values given by the zeroes of the denominator in $C_{l}$ (的). The characteristic spacing between these poles is given by the scale $L / R^{2}$ discussed earlier; asymptotically the spectrum is given by $q \sim n \pi L / R^{2}$ for integer $n$. From the AdS perspective the presence of these poles is understood by noting that the AdS plus shell physics is effectively that of a cavity; the origin is regular, and there is a growing potential at infinity. This geometry will have normal modes, and the poles in the propagator occur at the corresponding frequencies. Note that as the radius of the shell goes to zero, the geometry approaches that of the charge- $N$ extremal three brane. Allowed modes for this form a continuum. One can see this limit directly in the above; in this limit the spacing between states vanishes.

Nonetheless, the $L \rightarrow 0$ limit raises a puzzle, as it isn't clear how this limit matches onto physics at $L=0$. Indeed, to an infalling observer, it might seem that these are very different configurations: for $L \approx 0$, the infalling observer would seem to have a very painful impact on the shell resulting from the large blueshift acquired on falling in, whereas the case $L=0$ results in a smooth horizon. Of course, for any finite $L>0$, conformal symmetry is broken in the boundary theory; from this perspective the limit isn't necessarily expected to be smooth.'

Another puzzle regards the emergence of the above sharp resonances. It is not clear how this behavior smoothly goes over into the expected $L=0$ behavior, and we furthermore do not understand their origin in the boundary description. One proposal is that the spectrum of resonances is a bound-state spectrum of the $W$-anti-W pairs

\footnotetext{
${ }^{4}$ We thank Juan Maldacena for a discussion on this point.
} 
mentioned earlier. At tree level, the lightest $\mathrm{W}$ states have masses $\sim g^{1 / 5} / R^{4 / 5}$, but tight binding effects could conceivably produce a spectrum with the spacing $\sim L / R^{2}$ found earlier.

We do not have a resolution to these puzzles, but part of the story certainly involves an important physical effect that we have neglected. A string impinging on a single D3-brane may be absorbed, converting into open string states on the brane. Therefore, when we send a wave into the shell geometry, it has a finite amplitude to be absorbed by the shell. In order to produce the resonant behavior responsible for the above poles, the wave must pass through the shell an infinite number of times, which it can't do without eventually being absorbed. In the presence of absorption the resonances are no longer infinitely sharp. We don't yet have an analytic calculation of this effect, but we will give some estimates of its magnitude.

To begin, recall that if a wave has a frequency $\omega$ at infinity in the full asymptotically flat solution (or in the pure AdS case, at $r=R$ ), it will acquire the large blueshift noted above and have a proper frequency $\omega_{p}=R \omega / L$ when it reaches the shell. For small $L$ this frequency is quite high even for moderate asymptotic frequencies. At low frequencies we'll use known results to estimate this absorption rate. But for $\omega_{p} \gtrsim 1$ in string units, one expects the absorption cross section to climb steeply as one encounters the increasing number of excited open string states that may be created on the brane. In this region it is difficult to make precise estimates.

For frequencies $\omega_{p}<1$, we will estimate the absorption using the results of [13]. Consider a plane wave of frequency $\omega_{p}$ incident on a planar configuration of branes with $n$ branes per unit area; for later correspondence with the shell case, we will take $n \sim N / R^{5}$. The incident wave can create long strings of length $\sim \omega_{p}$ connecting the branes. This means that we should think of a single cluster of branes with radius $\sim \omega_{p}$ as a single absorber, and we will approximate the absorption cross section for the cluster by the result for an equal number of coincident branes. This cluster has $N_{e f f} \sim n \omega_{p}^{5}$ branes. For $\omega_{p}<1$ the effective radius $R_{e f f}=\left(g N_{e f f}\right)^{1 / 4}$ satisfies $\omega_{p} R_{e f f} \ll 1$, and so the cross-section, which grows like $\left(\omega_{p} R_{e f f}\right)^{8+4 l} / \omega_{p}^{5}$ for the $l$ th partial wave [14, is dominated by the case $l=0$. Thus the cross-section for the cluster is

$$
\sigma_{c l} \sim g^{2} N_{e f f}^{2} \omega_{p}^{3} \sim g^{2} N^{2} \frac{\omega_{p}^{10}}{R^{10}} \omega_{p}^{3} \sim \frac{\omega_{p}^{13}}{R^{2}} .
$$

Assuming that the absorption cross-sections for the different clusters add, this means that the sheet of three branes has an absorption cross-section per unit area, or "opacity" given by

$$
\frac{1}{\omega_{p}^{5}} \sigma_{c l} \sim \frac{\omega_{p}^{8}}{R^{2}} .
$$

So long as the cluster size is small compared to the radius of the sphere, this planar 
analysis should be a good approximation for spherically symmetric shells. Since the cluster size $\sim \omega_{p}$ is less than the string scale, this is true in our case.

We can describe this absorption with modified boundary conditions at the plane. We must always require that the field itself is continuous at the plane, but the absorption can be accounted for by introducing a discontinuity in the normal derivative:

$$
\left[n^{\mu} \nabla_{\mu} \phi\right]=-\alpha \phi
$$

with complex $\alpha$. We use here a notation $\left.[\Psi] \equiv \Psi\right|_{r=l+\epsilon}-\left.\Psi\right|_{r=l-\epsilon}$. From the point of view of the equations of motion, we are adding a complex potential $\alpha \delta(r-L)$ to the previous homogeneous equation. The jump in the probability flux is

$$
\left[n^{\mu} J_{\mu}\right] / \omega_{p}=-2 \operatorname{Im}(\alpha)|\phi|^{2} / \omega_{p}
$$

We will therefore take

$$
\operatorname{Im}(\alpha) \sim \frac{\sigma_{c l}}{\omega_{p}{ }^{4}}
$$

The precise value of the real part will not affect the qualitative conclusions. The solutions in the interior and exterior regions are still given by (4) and (5), but the new matching conditions will give a different value for $C_{l}$, namely

$$
C_{l}=-\frac{J_{l+2}(s) N_{l+2}^{\prime}(s)+N_{l+2}(s) J_{l+2}^{\prime}(s)-\frac{\alpha L}{q R} J_{l+2}(s) N_{l+2}(s)}{2 J_{l+2}(s) J_{l+2}^{\prime}(s)-\frac{\alpha L}{q R} J_{l+2}^{2}(s)} .
$$

Furthermore, the action no longer reduces to just a surface term at infinity, as the surface terms induced on $r=L$ by the bulk action inside and outside the shell will fail to cancel as a result of the discontinuity (13). The action will be

$$
I=\frac{1}{2} \int_{z=0} d \Sigma^{\mu} \phi \nabla_{\mu} \phi-\frac{1}{2} \int_{z=R^{2} / l} d \Sigma^{\mu} \phi\left[\nabla_{\mu} \phi\right] .
$$

This modifies the result (10) to give a two-point function

$$
\begin{aligned}
\left\langle\mathcal{O}_{l m}(\omega, k) \mathcal{O}_{l^{\prime} \vec{m}^{\prime}}\left(\omega^{\prime}, k^{\prime}\right)\right\rangle= & \frac{i R^{8}}{(l+1) !} \delta_{l l^{\prime}} \delta_{\vec{m} \vec{m}^{\prime}}(2 \pi)^{4} \delta^{4}\left(k-k^{\prime}\right)\left(\frac{q}{2}\right)^{2 l+4} \\
& \times\left(\frac{l+4}{(l+2) !}\left\{2 \ln (q / 2)-[\psi(1)+\psi(l+3)]+2 /(l+4)+\pi C_{l}\right\}\right. \\
& \left.+\alpha R \frac{\pi^{2}}{(l+1) !}\left[N_{l+2}(s)+C_{l} J_{l+2}(s)\right]^{2}\right),
\end{aligned}
$$

with $C$ given in (16). p

\footnotetext{
${ }^{5}$ This approach to the calculation of the two-point function involves a surface term at the shell. Nonetheless, the boundary two-point function should still be the scaled limit of the bulk two-point function; the latter also includes a contribution arising from absorption at the shell.
} 
Now $\alpha$ is complex, so its presence in the denominator of $C_{l}$ will move half of the poles in this expression off the real axis a distance proportional to the absorption by the shell. The remaining poles should be moved to complex values once the finite thickness of the shell is accounted for. This decay of would-be normalisable modes via absorption by the shell is described from the field theory point of view as the decay of a composite object into pairs of gauge bosons. The fractional width of the resonances are easily estimated to be $\mathcal{O}\left(\omega^{7} R^{4} / L^{7}\right)$. For low frequencies the resonances remain extremely sharp and the last term on the right hand side of eq. (18) is subdominant. At increasing frequency or equivalently decreasing $L$ they broaden, and we expect these estimates to break down and the shell to become opaque once the proper frequency at the shell reaches the string scale. One might hope to match this absorption onto the extremal three brane case at $L=0$, but this remains a puzzle. Since the seperation between the resonances is larger than their width up to $\omega_{p} \sim 1$, we can only hope to recover the continuum characteristic of the $L=0$ solution above this scale; note that because of the large blueshift, this corresponds to $\omega \sim L / R$, and this energy scale is still lower than the Higgs scale from the gauge theory point of view.

We can also calculate the Wilson loops in the boundary gauge theory. We expect that as the scale of the Wilson loop exceeds $R^{2} / L$, the string worldsheet spanning the loop will pass from being some minimal surface in the AdS part of the geometry to being stretched straight from the Wilson loop to the D3-brane shell. In the original pure AdS calculation, the energy of the minimal surface between two static quarks seperated by $d$ was found to be 15

$$
E=-\frac{4 \pi^{2} R^{2}}{\Gamma(1 / 4)^{4} d}
$$

The energy of a pair of strings stretching from the quarks to the surface $r=L$ is

$$
E=-\frac{L}{\pi}
$$

because it is less than the energy of the strings stretching to $r=0$ which were used to fix the zero of energy in this calculation by this amount. Hence we see that if $d>R^{2} / L$, the straight strings will have less energy than the minimal surface. When $d \sim R^{2} / L$, the minimal surface stretches down to $r_{0} \sim L$. Thus, we see that there is complete screening of the quark-anti quark potential for $d>R^{2} / L$, as in the calculations for disks in [5, 6].

A disappointing feature of our results is that we have been unable to access the flat space region. For the two-point function, we can only calculate in a regime at energies below the Higgs scale, and we expect that at higher frequencies the shell will become effectively opaque, and will prevent the probes from entering the flat region. Similarly, in the Wilson loop calculation, when the loop is sufficiently large for the string spanning it to reach the shell, it simply breaks and terminates on the shell. Thus, it seems we cannot learn anything about flat space holography from this solution. This failure may 
be regarded as having been prefigured by the calculation in [16], where it was observed that the energy/distance relation becomes flat in the region of interest.

To summarize, the spherically symmetric shell (1) provides an extremely simple example of a supergravity solution corresponding to the Coulomb branch of the gauge theory. However, because of the high degree of symmetry of the solution, none of the one-point functions of operators dual to supergravity fields can have non-zero values; in particular, the non-zero Higgs vevs are related to expectation values for operators whose dimensions are not protected, corresponding to massive string fields on the gravity side. It would be interesting if these expectation values could be estimated from the gravity side; however, lacking a better control of the stringy details, we have had to focus on studying this solution more indirectly, through the supergravity two-point function and Wilson loop expectation values. Our desire to treat the shell realistically, as being composed of D3-branes, forces us to face a complication in the two-point function calculations: the boundary conditions at the shell must be chosen to express the absorption of incident wavepackets onto the 3-branes. We can thus only calculate the two-point function at very low frequencies. At high frequencies one might hope to use a WKB approximation, but lack of detailed understanding of absorption presents an obstacle. Taking absorption into account gives the poles in the two-point function an imaginary part, indicating that they correspond to unstable resonances decaying into gauge fields. The calculation of the Wilson loop is more straightforward, and indicates a sharp cutoff in the quark-anti-quark potential at separations $d \sim R^{2} / L$, beyond which there is complete screening of the potential, as in [5, 6]. Both effects can be interpreted as part of the transition from perturbative D-brane behavior to that of a large extremal black brane. It will be interesting to understand such crossover phenomena in more detail.

\section{Acknowledgments}

It is a pleasure to thank S. Gubser, A. Hashimoto, S. Kachru, J. Maldacena, R. Myers, H. Ooguri, J. Polchinski, and W. Taylor for discussions. S.G. would like to particularly thank the theory group at Berkeley and Lawrence Berkeley Labs, the Institute for Theoretical Physics and Center for Mathematical Physics at the University of Amsterdam, and the organizers of the Strings '99 conference, where parts of this work was carried out. S.G. is supported in part by DOE contract DE-FG-03-91ER40618, and S.F.R. by NSF grant PHY95-07065.

\section{References}

[1] J. Maldacena, "The large N limit of superconformal field theories and supergravity," Adv. Theor. Math. Phys. 2 (1998) 231, hep-th/9711200. 
[2] S. S. Gubser, I. R. Klebanov, and A. M. Polyakov, "Gauge theory correlators from noncritical string theory," Phys. Lett. B428 (1998) 105, hep-th/9802109.

[3] E. Witten, "Anti-de Sitter space and holography," Adv. Theor. Math. Phys. 2 (1998) 253, hep-th/9802150.

[4] P. Kraus, F. Larsen, and S. P. Trivedi, "The Coulomb branch of gauge theory from rotating branes," JHEP 03 (1999) 003, hep-th/9811120.

[5] D. Z. Freedman, S. S. Gubser, K. Pilch, and N. P. Warner, "Continuous distributions of D3-branes and gauged supergravity," hep-th/9906194.

[6] A. Brandhuber and K. Sfetsos, "Wilson loops from multicenter and rotating branes, mass gaps and phase structure in gauge theories," hep-th/9906201.

[7] R. Kallosh and A. Rajaraman, "Vacua of M theory and string theory," Phys. Rev. D 58, 125003 (1998) hep-th/9805041.

[8] U. H. Danielsson, E. Keski-Vakkuri, and M. Kruczenski, "Spherically collapsing matter in AdS, holography, and shellons," hep-th/9905227.

[9] V. Balasubramanian and S. F. Ross, "Holographic particle detection," hep-th/9906226.

[10] I. Chepelev and R. Roiban, "A note on correlation functions in AdS(5) / SYM(4) correspondence on the Coulomb branch," hep-th/9906224.

[11] J. Polchinski, private communication.

[12] D.Z. Freedman, S.D. Mathur, A. Matusis and L. Rastelli, "Correlation functions in the CFT(d) / AdS $(\mathrm{d}+1)$ correspondence," Nucl. Phys. B546, 96 (1999) hep-th/9804058.

[13] I. R. Klebanov, "World volume approach to absorption by nondilatonic branes," Nucl. Phys. B496 (1997) 231, hep-th/9702076.

[14] O. Aharony, S.S. Gubser, J. Maldacena, H. Ooguri and Y. Oz, "Large N field theories, string theory and gravity," hep-th/9905111.

[15] J. Maldacena, "Wilson loops in large N field theories," Phys. Rev. Lett. 80 (1998) 4859, hep-th/9803002.

[16] A. Hashimoto, "Holographic description of D3-branes in flat space," hep-th/9903227. 